\title{
Traditional food diversity predicts dietary quality for the Awajún in the Peruvian Amazon
}

\author{
ML Roche ${ }^{1,2}$, HM Creed-Kanashiro ${ }^{3}$, I Tuesta ${ }^{4}$ and HV Kuhnlein 1,2,* \\ ${ }^{1}$ Centre for Indigenous Peoples' Nutrition and Environment (CINE), McGill University, Macdonald Campus, \\ 21111 Lakeshore Road, Ste. Anne de Bellevue, Quebec, Canada, H9X 3V9: ${ }^{2}$ School of Dietetics and Human \\ Nutrition, McGill University, Montreal, Canada: ${ }^{3}$ Instituto de Investigación Nutricional (IIN), Lima, Peru: \\ ${ }^{4}$ Organización de Desarollo de las Comunidades Fronterizas del Cenepa (ODECOFROC), Rio Cenepa, \\ Amazonas, Peru
}

Submitted 6 June 2006: Accepted 15 May 2007: First published online 5 July 2007

\begin{abstract}
Objective: Our goal was to assess the potential for evaluating strengths of the Awajún traditional food system using dietary assessment, a traditional food diversity score and ranking of local foods.

Design: The method was used for dietary data obtained from mothers and children in the Awajún culture of the Peruvian Amazon where $>90 \%$ of the dietary energy is derived from local, traditional food. Traditional food diversity scores were calculated from repeat 24-hour recalls. Group mean intakes of energy, fat, protein, iron, vitamin $\mathrm{A}$ and vitamin $\mathrm{C}$ from each food item were used to rank foods by nutrient contribution.

Setting: The study took place in six remote communities along the lower Cenepa River in the Amazonas District of Peru, South America.

Subjects: Dietary data were collected from 49 Awajún mothers and 34 children aged 3-6 years, representative of the six communities.

Results: Higher traditional food diversity was associated with greater protein, fibre, vitamin and mineral intakes when controlling for energy (partial correlations $=0.37$ to 0.64 ). Unique sources for iron, total vitamin A and vitamin $\mathrm{C}$ were found in the Awajún traditional food system.

Conclusions: A traditional food diversity score was a useful tool for predicting nutrient adequacy for the Awajún. Promotion of the Awajún traditional food system should focus on dietary diversity and unique nutrient-dense local foods.
\end{abstract}

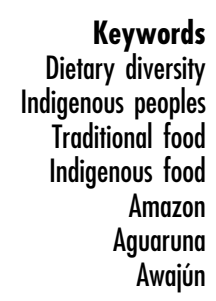

A traditional food system of indigenous peoples is defined as being comprised of all acceptable foods provided by the natural resources of a particular cultural group; this can include indigenous and introduced cultivated plants and animals ${ }^{1}$. In addition to supporting health, being affordable and often protective of the ecosystem, traditional food systems contain great cultural value for indigenous peoples ${ }^{2}$. To promote and protect the traditional food systems of indigenous peoples, local resources must be understood, with a need to document food diversity and understand its relationship to the health of communities ${ }^{3}$.

Dietary diversity is often promoted in guidelines for healthy diets ${ }^{4}$. It can simply be defined as the number of unique foods, species or food groups within an individual or group diet. This measure of variety is usually calculated by a simple count of unique foods or food groups consumed over a given reference period ${ }^{5}$, with reference periods ranging from 1 to 15 days $^{6}$. Recent studies aimed at validating dietary diversity against nutrient adequacy in developing countries ${ }^{7-9}$ have shown a positive relationship that has been documented in industrialised nations ${ }^{10}$. When there are difficulties in calculating individual intakes due to shared meals or lack of specific nutrient data for local foods, a dietary diversity score calculated from a dietary recall may be an appropriate predictor for evaluating dietary adequacy ${ }^{8}$.

The Awajún† live in the remote hills of the northwestern Amazon of Peru, subsisting on a cassava-based diet complemented by hunting, gathering and fishing ${ }^{11}$. In a nutritional anthropology study reported in 1977, Berlin and Markell reported a generally healthy population (good nutritional status) with an adequate household

$\uparrow$ Awajún is the preferred identity for this group of Indigenous People, who are also known as the Aguaruna. 
food intake ${ }^{11}$. Here we report dietary research with the Awajún thirty years later, and explore the relationship between dietary diversity of traditional foods and nutrient adequacy. We highlight unique local foods for Awajún women and children living in several communities near the Rio (River) Cenepa.

\section{Methods}

\section{Study communities}

The study took place in six communities in the lower Cenepa River area in the Amazonas District of Peru, South America: Cocoaushi [Annex of Wawaim (638 people, 101 families)], Mamayaque (350 people, 65 families), Nuevo Tutino (110 people, 22 families), Tuutin (350 people, 61 families), Pagki (60 people, 10 families) and Nuevo Kanam [Annex of Tuutin (217 people, 47 families) ${ }^{12}$. The study took place during the months of April and May 2004, known by the Awajún as the season of heavy rains and scarce fishing. Key informant interviews and focus groups established the list of traditional foods, and their pattern of use, that formed the basis of development of a food composition table for the area. All women with children 3-6 years of age were invited to participate in the study. Usually, there was one woman per household, and when there were two or more children between 3 and 6 years old, one child was randomly selected. Participation rates were approximately 80\%. Lack of time for the study and scepticism towards it, as well as the need to care for a family member, were given as reasons for not participating. Follow-up with non-participants by an Awajún community health promoter indicated no obvious participation bias, and the final sample was judged to be representative of the communities in terms of livelihoods and family structure.

\section{Dietary recalls}

From March to April 2004, two standardised 24-hour dietary recalls were obtained from each of 49 mothers and 34 children with a separation of 3-4 days between repeat recalls ${ }^{13}$. The interviewers were trained and they were assisted by an Awajún interpreter. To improve quality of dietary recalls ${ }^{14}$, several samples of cassava (Manibot esculenta), sachapapa (Dioscorea trifida), bananas and plantains with known weights were used as references during interviews. Cups, plates and bowls, and pinig (bowl for drinking) found in the communities were also used as references for quantifying food consumption. Community focus groups helped establish local portion sizes, preparation methods and cultural acceptability of food. Mothers reported foods consumed for themselves and for their children.

Dietary recall data were reviewed and checked for accuracy, and food codes were added that corresponded to the Instituto de Investigación Nutricional (IIN) food composition table of (raw) Peruvian foods. With respect to the vitamin A content of foods, the Peruvian food composition table used retinol equivalents (RE) at the time of this study; 11 food items were added from other tables using the international literature for food composition $^{15-18}$. Information from the Peruvian food composition tables or the nutrient labels was taken at the time of the study for the small number of foods imported into these communities.

Daily total intake for each nutrient for each individual was obtained by averaging the two 24-hour recalls from non-consecutive days. No adjustments for bioavailability of nutrients were made as this was unknown for most of these foods.

\section{Traditional food diversity}

A traditional food diversity score (TFDS) was calculated by assigning a 1 point value to each unique local food in the diet reported in the two 24-hour recalls, and an individual's TFDS was the sum of points given. These traditional food items included all foods obtained through cultivating, hunting, gathering, fishing and raising animals. An individual's TFDS was then related to the mean daily nutrient intake calculated from the two 24-hour recalls using partial correlations controlling for energy intake of the women and children. Statistical analysis was performed with SPSS 11.0 for Windows (SPSS Inc., 2001).

\section{Nutrient contributions of local foods}

The 10 most significant contributors of overall energy, protein and fat, total iron, vitamin $\mathrm{C}$ and vitamin A (RE) were determined by ranking the mean amount of nutrient provided. Ranks were made independently for mothers' and children's diets.

\section{Etbics}

Ethics approval was given by the Faculty of Agricultural and Environmental Sciences Committee on Human Research Ethics at McGill University as well as from the Institutional Ethics Board for the IIN, in Lima, Peru. This study followed the guidelines for participatory health research with indigenous peoples established by the World Health Organization and the Centre for Indigenous Peoples' Nutrition and Environment (CINE) ${ }^{19}$. A community research agreement was in effect with the local village authority, the Organizacion de Desarrollo de las Communidades Fronterizas del Cenepa (ODECOFROC), the IIN and CINE. Verbal informed consent was obtained from mothers for both themselves and their children. 


\section{Results}

\section{Traditional food diversity}

The TFDS ranged from 2 to 20 for the women, with a median of 9 and a mean ( \pm standard deviation, SD) of $9.5 \pm 3.5$. For children the TFDS ranged from 2 to 17 with a median of 8 and mean ( \pm SD) of $8.7 \pm 3.6$. Higher TFDS was correlated with higher nutrient intakes for many vitamins and minerals for both women and children (Table 1). Greater TFDS was associated with higher

Table 1 Partial correlation of traditional food diversity scores and nutrient intakes controlling for energy for Rio Cenepa Awajún women and children

\begin{tabular}{lll}
\hline Nutrient & Women $(n=49)$ & Children $(n=35)$ \\
\hline Protein & $0.44^{\star \star}$ & $0.53^{\star \star}$ \\
Fat & $0.31(P=0.30)$ & $0.18(P=0.30)$ \\
Fibre & $0.66^{\star \star}$ & $0.37^{\star}$ \\
Calcium & $0.51^{\star \star}$ & $0.30(P=0.08)$ \\
Phosphorus & $0.47^{\star \star}$ & $0.29(P=0.09)$ \\
Iron & $0.49^{\star \star}$ & $0.39^{\star}$ \\
Zinc & $0.20(P=0.16)$ & $0.25(P=0.16)$ \\
Thiamin & $0.62^{\star \star}$ & $0.64^{\star \star}$ \\
Riboflavin & $0.49^{\star \star}$ & $0.38^{\star}$ \\
Niacin & $0.38^{\star \star}$ & $0.20(P=0.29)$ \\
Vitamin C & $0.40^{\star \star}$ & $0.26(P=0.13)$ \\
Folate & $0.38^{\star \star}$ & $0.17(P=0.35)$ \\
Total vitamin A & $0.43^{\star \star}$ & $0.42^{*}$ \\
\hline
\end{tabular}

${ }^{*} P<0.05$ (two-tailed); ${ }^{* *} P<0.01$ (two-tailed). dietary protein, fibre, iron, thiamin, riboflavin and vitamin A among women and children. Higher dietary calcium, phosphorus, niacin, vitamin $\mathrm{C}$ and folate were correlated with greater diversity scores for women (partial correlations $=0.37$ to 0.64 ). There was no relationship between TFDS and total energy intake in either group.

\section{Nutrient contributions of local foods}

The high-dietary-energy sources of cassava, banana, sachapapa (a tuber) and masato (thick traditional beverage made from pre-masticated cassava) were consumed daily in the research period by the majority of the population in large amounts (daily average consumption of over $1.0 \mathrm{~kg}$ of cassava by women and $0.65 \mathrm{~kg}$ by children) (Table 2) and consequently provided substantial nutrients. More nutrient-dense foods were successful in providing protein, fat, vitamins $\mathrm{A}$ and $\mathrm{C}$, iron and zinc to the population, even though consumed in smaller portions by fewer individuals (Tables 3-7). This is highlighted by locally grown raw peanuts which were consumed in average amounts of $163 \pm 145 \mathrm{~g}$ by five women (Table 4) and $116 \pm 107 \mathrm{~g}$ by five children, yet provided twice the fat for women and five times the fat for children in comparison with cassava, consumed in average amounts of $1271 \pm 879 \mathrm{~g}$ by all 49 women and $647 \pm 504 \mathrm{~g}$ by all 35 children. The rank orders of the top

Table 2 Top 10 foods contributing energy for Rio Cenepa Awajún women and children

\begin{tabular}{|c|c|c|c|}
\hline \multicolumn{4}{|l|}{ Women $(n=49)$} \\
\hline Food & $\begin{array}{l}\text { Total population/mean } \pm \text { SD individual } \\
\text { daily energy }(\mathrm{kcal})+\end{array}$ & $\begin{array}{l}\text { Mean } \pm \text { SD daily food } \\
\text { consumption }(\mathrm{g}) \dagger\end{array}$ & $\begin{array}{c}\text { Days in 24-hour recall [max 98] } \\
\text { (no. of women) }\end{array}$ \\
\hline Cassava & $95806 / 1955 \pm 1094$ & $1271 \pm 879$ & $93(49)$ \\
\hline Banana & $14348 / 359 \pm 310$ & $629 \pm 439$ & $55(40)$ \\
\hline Masato & $9572 / 309 \pm 217$ & $1176 \pm 669$ & $44(31)$ \\
\hline Sachapapa & $8918 / 594 \pm 527$ & $838 \pm 790$ & $19(15)$ \\
\hline Plantain & $6987 / 279 \pm 175$ & $340 \pm 205$ & $27(25)$ \\
\hline Sugar cane & $3827 / 201 \pm 214$ & $406 \pm 327$ & $23(19)$ \\
\hline Raw peanuts & $3187 / 637 \pm 520$ & $163 \pm 145$ & $7(5)$ \\
\hline White rice & $3133 / 285 \pm 154$ & $134 \pm 61$ & $13(11)$ \\
\hline Peach palm masato & $2763 / 395 \pm 459$ & $1470 \pm 1790$ & $8(7)$ \\
\hline Taro & $2518 / 280 \pm 150$ & $548 \pm 295$ & $9(9)$ \\
\hline \multicolumn{4}{|l|}{ Children $(n=35)$} \\
\hline Food & $\begin{array}{l}\text { Total population/mean } \pm \text { SD individual } \\
\text { daily energy }(\mathrm{kcal})+\end{array}$ & $\begin{array}{l}\text { Mean } \pm \text { SD daily food } \\
\text { consumption }(\mathrm{g}) \dagger\end{array}$ & $\begin{array}{c}\text { Days in 24-hour recall [max 70] } \\
\text { (no. of children) }\end{array}$ \\
\hline Cassava & $33558 / 959 \pm 627$ & $647 \pm 504$ & $64(35)$ \\
\hline Banana & $9865 / 318 \pm 247$ & $495 \pm 365$ & $48(31)$ \\
\hline Sachapapa & $8702 / 669 \pm 720$ & $863 \pm 983$ & $18(13)$ \\
\hline White rice & $5136 / 321 \pm 211$ & $130 \pm 65$ & $22(16)$ \\
\hline Plantain & $4639 / 258 \pm 181$ & $291 \pm 193$ & $21(18)$ \\
\hline Masato & $3157 / 158 \pm 79$ & $569 \pm 262$ & $30(20)$ \\
\hline Raw peanuts & $2595 / 519 \pm 400$ & $116 \pm 107$ & $8(5)$ \\
\hline Yellow beans & $2124 / 177 \pm 89$ & $80 \pm 31$ & $16(12)$ \\
\hline Taro & $2022 / 337 \pm 328$ & $566 \pm 600$ & $7(6)$ \\
\hline Sweet potato & $1751 / 583 \pm 571$ & $755 \pm 916$ & $4(3)$ \\
\hline
\end{tabular}

SD - standard deviation.

t Means calculated using as $n$ the number of women or children who consumed the food (no. of women or children). 
Table 3 Top 10 foods contributing protein for Rio Cenepa Awajún women and children

Women $(n=49)$

\begin{tabular}{|c|c|c|c|}
\hline Food & $\begin{array}{c}\text { Total population/mean } \pm S D \text { individual } \\
\text { daily protein }(\mathrm{g}) \dagger\end{array}$ & $\begin{array}{l}\text { Mean } \pm \text { SD daily food } \\
\text { consumption }(\mathrm{g})+\end{array}$ & $\begin{array}{c}\text { Days in 24-hour recall [max 98] } \\
\text { (no. of women) }\end{array}$ \\
\hline Cassava & $473 / 10 \pm 5$ & $1271 \pm 879$ & $93(49)$ \\
\hline Armadillo & $261 / 29 \pm 14$ & $180 \pm 104$ & $10(9)$ \\
\hline Banana & $260 / 7 \pm 5$ & $629 \pm 439$ & $55(40)$ \\
\hline Chicken & $159 / 17 \pm 9$ & $174 \pm 88$ & $14(14)$ \\
\hline Sachapapa & $144 / 10 \pm 8$ & $838 \pm 790$ & $19(15)$ \\
\hline Raw peanuts & $138 / 27 \pm 22$ & $163 \pm 145$ & $7(5)$ \\
\hline Turkey & $124 / 41 \pm 28$ & $164 \pm 61$ & $4(3)$ \\
\hline Evaporated milk & $101 / 8 \pm 4$ & $191 \pm 175$ & $15(8)$ \\
\hline Palm heart & $79 / 7 \pm 7$ & $387 \pm 420$ & $14(13)$ \\
\hline White rice & $65 / 7 \pm 4$ & $134 \pm 61$ & $13(11)$ \\
\hline \multicolumn{4}{|l|}{ Children $(n=35)$} \\
\hline Food & $\begin{array}{l}\text { Total population/mean } \pm \text { SD individual } \\
\text { daily protein }(\mathrm{g}) \dagger\end{array}$ & $\begin{array}{l}\text { Mean } \pm \text { SD daily food } \\
\text { consumption }(\mathrm{g})+\end{array}$ & $\begin{array}{c}\text { Days in 24-hour recall [max 70] } \\
\text { (no. of children) }\end{array}$ \\
\hline Banana & $179 / 6 \pm 4$ & $495 \pm 365$ & $48(31)$ \\
\hline Cassava & $166 / 5 \pm 3$ & $647 \pm 504$ & $64(35)$ \\
\hline Yellow beans & $145 / 12 \pm 6$ & $80 \pm 31$ & $16(12)$ \\
\hline Sachapapa & $140 / 11 \pm 12$ & $863 \pm 983$ & $18(13)$ \\
\hline White rice & $118 / 7 \pm 5$ & $130 \pm 65$ & $22(16)$ \\
\hline Raw peanuts & $112 / 22 \pm 17$ & $116 \pm 107$ & $8(5)$ \\
\hline Chicken & $95 / 11 \pm 6$ & $109 \pm 47$ & $13(10)$ \\
\hline Turkey & $91 / 30 \pm 25$ & $208 \pm 146$ & $4(3)$ \\
\hline Evaporated milk & $82 / 7 \pm 7$ & $279 \pm 212$ & $10(10)$ \\
\hline Palm heart & $58 / 5 \pm 6$ & $282 \pm 325$ & 12 (12) \\
\hline
\end{tabular}

SD - standard deviation.

t Means calculated using as $n$ the number of women or children who consumed the food (no. of women or children).

Table 4 Top 10 foods contributing fat for Rio Cenepa Awajún women and children

\begin{tabular}{|c|c|c|c|}
\hline \multicolumn{4}{|l|}{ Women $(n=49)$} \\
\hline Food & $\begin{array}{l}\text { Total population/mean } \pm \text { SD individual } \\
\text { daily fat }(\mathrm{g})+\end{array}$ & $\begin{array}{l}\text { Mean } \pm \text { SD daily food } \\
\text { consumption }(\mathrm{g}) \dagger\end{array}$ & $\begin{array}{c}\text { Days in 24-hour recall [max 98] } \\
\text { (no. of women) }\end{array}$ \\
\hline $\begin{array}{l}\text { Raw peanuts } \\
\text { Macambo seeds } \\
\text { Evaporated milk } \\
\text { Sachapapa } \\
\text { Cassava } \\
\text { Chicken } \\
\text { Sachamango } \\
\text { Turkey } \\
\text { Banana } \\
\text { Armadillo }\end{array}$ & $\begin{array}{c}275 / 55 \pm 45 \\
188 / 23 \pm 24 \\
125 / 9 \pm 4 \\
120 / 8 \pm 7 \\
119 / 3 \pm 1 \\
113 / 13 \pm 7 \\
78 / 17 \pm 7 \\
71 / 6 \pm 4 \\
52 / 1 \pm 1 \\
49 / 5 \pm 3\end{array}$ & $\begin{array}{c}163 \pm 145 \\
69 \pm 77 \\
191 \pm 175 \\
838 \pm 790 \\
1271 \pm 879 \\
174 \pm 88 \\
171 \pm 79 \\
164 \pm 61 \\
629 \pm 439 \\
180 \pm 104\end{array}$ & $\begin{aligned} & 7(5) \\
& 10(8) \\
& 15(8) \\
& 19(15) \\
& 93(49) \\
& 14(14) \\
& 5(5) \\
& 4(3) \\
& 55(40) \\
& 10(9)\end{aligned}$ \\
\hline \multicolumn{4}{|l|}{ Children $(n=35)$} \\
\hline Food & $\begin{array}{l}\text { Total population/mean } \pm \text { SD individual } \\
\text { daily fat }(\mathrm{g}) \dagger\end{array}$ & $\begin{array}{l}\text { Mean } \pm \text { SD daily food } \\
\text { consumption }(\mathrm{g}) \dagger\end{array}$ & $\begin{array}{c}\text { Days in 24-hour recall [max 70] } \\
\text { (no. of children) }\end{array}$ \\
\hline $\begin{array}{l}\text { Raw peanuts } \\
\text { Sachapapa } \\
\text { Evaporated milk } \\
\text { Macambo seeds } \\
\text { Chicken } \\
\text { Sachamango } \\
\text { Cassava } \\
\text { Canned tuna } \\
\text { Banana } \\
\text { Suri }\end{array}$ & $\begin{array}{c}224 / 45 \pm 35 \\
117 / 9 \pm 10 \\
97 / 8 \pm 7 \\
93 / 23 \pm 19 \\
87 / 12 \pm 7 \\
80 / 13 \pm 10 \\
42 / 1 \pm 1 \\
37 / 3 \pm 1 \\
36 / 1 \pm 1 \\
29 / 7 \pm 3\end{array}$ & $\begin{aligned} 116 & \pm 107 \\
863 & \pm 983 \\
279 & \pm 212 \\
68 & \pm 41 \\
109 & \pm 47 \\
125 & \pm 84 \\
647 & \pm 504 \\
19 & \pm 8 \\
495 & \pm 365 \\
38 & \pm 15\end{aligned}$ & $\begin{aligned} 8 & (5) \\
18 & (13) \\
10 & (10) \\
5 & (4) \\
13 & (10) \\
7 & (6) \\
64 & (35) \\
19 & (13) \\
48 & (31) \\
4 & (4)\end{aligned}$ \\
\hline
\end{tabular}

SD - standard deviation.

† Means calculated using as $n$ the number of women or children who consumed the food (no. of women or children). 
Table 5 Top 10 foods contributing iron for Rio Cenepa Awajún women and children

Women $(n=49)$

\begin{tabular}{|c|c|c|c|}
\hline Food & $\begin{array}{l}\text { Total population/mean } \pm \text { SD individual } \\
\text { daily iron }(\mathrm{mg}) \dagger\end{array}$ & $\begin{array}{l}\text { Mean } \pm \text { SD daily food } \\
\text { consumption }(\mathrm{g}) \dagger\end{array}$ & $\begin{array}{c}\text { Days in 24-hour recall [max 98] } \\
\text { (no. of women) }\end{array}$ \\
\hline Cassava & $296 / 6 \pm 3$ & $1271 \pm 879$ & $93(49)$ \\
\hline Masato & $155 / 5 \pm 4$ & $1176 \pm 669$ & $44(31)$ \\
\hline Banana & $104 / 3 \pm 2$ & $629 \pm 439$ & $55(40)$ \\
\hline Armadillo & $98 / 11 \pm 5$ & $180 \pm 104$ & $10(9)$ \\
\hline Sachapapa & $56 / 4 \pm 3$ & $838 \pm 790$ & $19(15)$ \\
\hline Maca & $52 / 52$ & 356 & $1(1)$ \\
\hline Palm heart & $46 / 4 \pm 4$ & $387 \pm 420$ & $14(13)$ \\
\hline Agouti & $41 / 8 \pm 1$ & $116 \pm 17$ & $5(5)$ \\
\hline Sugar cane & $33 / 2 \pm 2$ & $406 \pm 327$ & 23 (19) \\
\hline Taro & $30 / 3 \pm 2$ & $548 \pm 295$ & $9(9)$ \\
\hline \multicolumn{4}{|c|}{ Children $(n=35)$} \\
\hline Food & $\begin{array}{l}\text { Total population/mean } \pm \text { SD individual } \\
\text { daily iron }(\mathrm{mg}) \dagger\end{array}$ & $\begin{array}{l}\text { Mean } \pm S D \text { daily food } \\
\text { consumption }(\mathrm{g})+\end{array}$ & $\begin{array}{c}\text { Days in 24-hour recall [max 70] } \\
\text { (no. of children) }\end{array}$ \\
\hline Cassava & $104 / 3 \pm 2$ & $647 \pm 504$ & $64(35)$ \\
\hline Banana & $72 / 2 \pm 2$ & $495 \pm 365$ & $48(31)$ \\
\hline Sachapapa & $55 / 4 \pm 5$ & $863 \pm 983$ & 18 (13) \\
\hline Masato & $51 / 3 \pm 1$ & $569 \pm 262$ & 30 (20) \\
\hline Yellow beans & $49 / 4 \pm 2$ & $80 \pm 31$ & $16(12)$ \\
\hline Palm heart & $29 / 2 \pm 3$ & $282 \pm 325$ & $12(12)$ \\
\hline Taro & $24 / 4 \pm 4$ & $566 \pm 600$ & $7(6)$ \\
\hline Cocona & $17 / 2 \pm 2$ & $151 \pm 164$ & $15(11)$ \\
\hline Suri & $16 / 4 \pm 2$ & $38 \pm 15$ & $4(4)$ \\
\hline Plantain & $15 / 1 \pm 1$ & $291 \pm 193$ & $21(18)$ \\
\hline
\end{tabular}

SD - standard deviation.

+ Means calculated using as $n$ the number of women or children who consumed the food (no. of women or children).

Table 6 Top 10 foods contributing vitamin C for Rio Cenepa Awajún women and children

\begin{tabular}{|c|c|c|c|}
\hline \multicolumn{4}{|l|}{ Women $(n=49)$} \\
\hline Food & $\begin{array}{l}\text { Total population/mean } \pm \text { SD individual } \\
\text { daily vitamin } C(\mathrm{mg}) \dagger\end{array}$ & $\begin{array}{l}\text { Mean } \pm \text { SD daily food } \\
\text { consumption }(\mathrm{g}) \dagger\end{array}$ & $\begin{array}{c}\text { Days in 24-hour recall [max 98] } \\
\text { (no. of women) }\end{array}$ \\
\hline Cassava & $18156 / 371 \pm 207$ & $1271 \pm 879$ & $93(49)$ \\
\hline Papaya & $1650 / 115 \pm 93$ & $629 \pm 351$ & $11(10)$ \\
\hline Masato & $1087 / 35 \pm 25$ & $1176 \pm 669$ & $44(31)$ \\
\hline Orange & $952 / 119 \pm 105$ & $229 \pm 125$ & $9(8)$ \\
\hline Banana & $744 / 19 \pm 16$ & $629 \pm 439$ & $55(40)$ \\
\hline Plantain & $478 / 19 \pm 12$ & $340 \pm 205$ & $27(25)$ \\
\hline Cassava leaves & $421 / 140 \pm 59$ & $97 \pm 40$ & $3(3)$ \\
\hline Peach palm masato & $288 / 41 \pm 48$ & $1470 \pm 1790$ & $8(7)$ \\
\hline Eep & $260 / 37 \pm 39$ & $22 \pm 26$ & $8(7)$ \\
\hline Sachapapa & $247 / 16 \pm 15$ & $838 \pm 790$ & 19 (15) \\
\hline \multicolumn{4}{|l|}{ Children $(n=35)$} \\
\hline Food & $\begin{array}{c}\text { Total population/mean } \pm \text { SD individual } \\
\text { daily vitamin } C(\mathrm{mg}) \dagger\end{array}$ & $\begin{array}{c}\text { Mean } \pm \text { SD daily food } \\
\text { consumption }(\mathrm{g}) \dagger\end{array}$ & $\begin{array}{c}\text { Days in 24-hour recall [max 70] } \\
\text { (no. of children) }\end{array}$ \\
\hline Cassava & $6360 / 182 \pm 119$ & $647 \pm 504$ & $64(35)$ \\
\hline Orange & $981 / 196 \pm 139$ & $266 \pm 162$ & $8(5)$ \\
\hline Papaya & $851 / 142 \pm 109$ & $510 \pm 395$ & $7(6)$ \\
\hline Banana & $511 / 16 \pm 13$ & $495 \pm 365$ & $48(31)$ \\
\hline Masato & $359 / 18 \pm 9$ & $569 \pm 262$ & $30(20)$ \\
\hline Plantain & $318 / 18 \pm 12$ & $291 \pm 193$ & $21(18)$ \\
\hline Sachapapa & $241 / 19 \pm 20$ & $863 \pm 983$ & $18(13)$ \\
\hline Sweet potato & $151 / 50 \pm 49$ & $755 \pm 916$ & $4(3)$ \\
\hline Sachamango & $121 / 20 \pm 15$ & $125 \pm 84$ & $7(6)$ \\
\hline Coco fruit & $118 / 39 \pm 22$ & $66 \pm 44$ & $4(3)$ \\
\hline
\end{tabular}

SD - standard deviation.

t Means calculated using as $n$ the number of women or children who consumed the food (no. of women or children).

$\ddagger$ Eep is a term to describe all green leaves (e.g. family Aracaceae, genus Philodendron). 
Table 7 Top 10 foods contributing total vitamin A for Rio Cenepa Awajún women and children

\begin{tabular}{|c|c|c|c|}
\hline \multicolumn{4}{|l|}{ Women $(n=49)$} \\
\hline Food & $\begin{array}{c}\text { Total population/mean } \pm S D \text { individual } \\
\text { daily vitamin } A(\mu g R E) \dagger\end{array}$ & $\begin{array}{l}\text { Mean } \pm \text { SD daily food } \\
\text { consumption }(g) t\end{array}$ & $\begin{array}{c}\text { Days in 24-hour recall [max 98] } \\
\text { (no. of women) }\end{array}$ \\
\hline Agouti & $13005 / 2601 \pm 398$ & $116 \pm 17$ & $5(5)$ \\
\hline Peach palm masato & $11581 / 1654 \pm 1925$ & $1470 \pm 1790$ & $8(7)$ \\
\hline Sweet potato & $11223 / 2245 \pm 2512$ & $618 \pm 754$ & $6(5)$ \\
\hline Paca & $6908 / 1151 \pm 736$ & $51 \pm 33$ & $6(6)$ \\
\hline Plantain & $5823 / 233 \pm 146$ & $340 \pm 205$ & 27 (25) \\
\hline Collared peccary & $3240 / 1620 \pm 1527$ & $48 \pm 46$ & $3(2)$ \\
\hline Evaporated milk & $2087 / 58 \pm 30$ & $191 \pm 175$ & $15(8)$ \\
\hline Armadillo & $2020 / 224 \pm 108$ & $180 \pm 104$ & $10(9)$ \\
\hline Banana & $1729 / 43 \pm 37$ & $629 \pm 439$ & $55(40)$ \\
\hline Peach palm fruit & $1507 / 251 \pm 232$ & $308 \pm 291$ & $7(6)$ \\
\hline \multicolumn{4}{|l|}{ Children $(n=35)$} \\
\hline Food & $\begin{array}{c}\text { Total population/mean } \pm S D \text { individual } \\
\text { daily vitamin } A(\mu g R E) \dagger\end{array}$ & $\begin{array}{l}\text { Mean } \pm \text { SD daily food } \\
\text { consumption }(\mathrm{g}) \dagger\end{array}$ & $\begin{array}{c}\text { Days in 24-hour recall [max 70] } \\
\text { (no. of children) }\end{array}$ \\
\hline Sweet potato & $9130 / 3043 \pm 2980$ & $755 \pm 916$ & $4(3)$ \\
\hline Paca & $3983 / 797 \pm 565$ & $35 \pm 25$ & $5(5)$ \\
\hline Plantain & $3866 / 215 \pm 150$ & $291 \pm 193$ & 21 (18) \\
\hline Agouti & $3713 / 1238 \pm 113$ & $55 \pm 5$ & $3(3)$ \\
\hline Peach palm masato & $3468 / 578 \pm 614$ & $587 \pm 623$ & $6(6)$ \\
\hline Evaporated milk & $1710 / 448 \pm 332$ & $279 \pm 212$ & $10(10)$ \\
\hline Banana & $1189 / 38 \pm 30$ & $495 \pm 365$ & $48(31)$ \\
\hline Chicken liver & $1172 / 1172$ & 69 & $1(1)$ \\
\hline Papaya & $655 / 109 \pm 84$ & $510 \pm 395$ & $7(6)$ \\
\hline Chicken egg & $607 / 67 \pm 42$ & $53 \pm 25$ & $12(9)$ \\
\hline
\end{tabular}

$\mathrm{SD}$ - standard deviation; RE - retinol equivalents.

t Means calculated using as $n$ the number of women or children who consumed the food (no. of women or children).

10 foods providing these nutrients for women and children are given in Tables 3-7. The amount of nutrients contributed (by those who consumed the food), the mean weight of food consumed and the frequency of reports in recalls emphasise the critical importance of these items to daily food intake and nutrition of Awajún women and children. Donated white rice, evaporated milk (not fortified), canned tuna (in oil), vegetable oil, dried yellow beans, oats and sugar (unfortified) were the only foods in these tables imported from outside sources.

Suri (larvae of the order Coleopterus) was the only animal source of iron which ranked in the top 10 for children, while agouti (Dasyprocta aguti; a small rodent) and armadillo (Tolypeutes mataco) ranked highly for women. Maca (orange tuber; Lepidium peruvianum Chacon) is the most iron-dense of the tubers and was the sixth greatest contributor of iron, yet was consumed by only one mother in a portion size of $356 \mathrm{~g}$. In addition to dietary staples other sources of iron for women were sugar cane, taro (Colocasia esculenta) and palm heart. Several palms were harvested (e.g. Bactris gasipaes, Astrocaryum chambira, Socratea exhorriza, Mauritia flexuosa and Euterpe precatoria). Beans, plantain, palm heart, taro and cocona (Solanum spp.) provided iron for children. Vitamin A was provided mainly by non-staple sources including agouti, peach palm masato (thick traditional beverage made from pre-masticated peach palm fruit, B. gasipaes), sweet potato (Ipomea batatas), paca (Cuniculus paca) and armadillo for both groups, as well as collared peccary (Tayassu tajacu) for mothers and, for children, chicken liver, chicken egg and papaya (Carica papaya). In both groups, evaporated milk contributed to vitamin A intakes.

Government-donated foods including white rice, beans, evaporated milk, canned tuna, vegetable oil, oats and sugar provided energy and nutrients for both women and children; however, these nutrients were also available in frequently consumed local foods.

\section{Discussion}

The nutritional importance of traditional food diversity during the study season was demonstrated through the relationship of greater nutrient intakes with higher TFDS for women and children. Various studies around the world have found positive associations between dietary diversity and nutritional intake, including among women and men in rural western Mali ${ }^{9}$, Korean adolescents ${ }^{20}$, and 10- to 18-year-olds in Tehran ${ }^{7}$ and urban Mali ${ }^{8}$. Most other food diversity studies have focused on overall dietary diversity, measured either by food groups or individual food items over a fixed period of time ${ }^{5}$. The TFDS was an appropriate measure for the Awajún perhaps because of the high traditional food content of 
the diet and the high quality of several of their traditional foods.

It has been suggested that caution must be taken in the promotion of dietary variety in some environments. The message of diversity could be wrongly interpreted and result in the consumption of many different low-nutrientdensity inexpensive foods, perpetuating the nutrition transition and its health consequences ${ }^{21}$. As an increasing variety and quantity of market foods may become available in the Cenepa region, community groups and leaders should promote the production and consumption of diverse, local, micronutrient-dense cultural foods and ensure the quality of food imported to the area, rather than simply encouraging overall dietary diversity. The TFDS could be a useful tool for assessing dietary quality of the Awajún in response to health promotion focusing on dietary variety.

Assessment of the dietary quality for women and children in the lower Cenepa River region indicated adequate intakes of energy, protein, fat, iron, zinc, vitamin $\mathrm{C}$ and vitamin A for women and 3-6-year-old children for the study season (reported elsewhere) ${ }^{22}$. The dietary intakes appeared to be adequate in the population studied. However, a limitation of this study was that we were unable to observe or weigh precisely the amount of food consumed by each individual; thus there may have been overestimation of intake through dietary recall in some cases because food was served communally and not on individual plates, or children did not eat all they were served. Nevertheless, the overreporting was most likely to have influenced quantity but not variety or proportions; thus we consider the relationships between nutrient intakes and diversity of the diet to be valid ${ }^{22}$. Here we report that the traditional food system of the Awajún in lower Cenepa River region offered several unique nutrient-dense food sources contributing to overall dietary sufficiency. Raw peanuts (Arachis bypogaeaa) were important for energy, protein and fat, with a portion size a fraction of that of cassava. In addition to cassava, other important tubers contributing energy as well as nutrients were sachapapa (a tuber), taro and sweet potato. Along with staple bananas, a variety of fruits contributed to nutrient intake, including papaya, sachamango (Grias peruviana), cocoa fruit (Theobroma cocoa), macambo seeds (Theobroma bicolor), peach palm (B. gasipaes) and Mauritian palm fruit (M. flexuosa).

Palms proved to be of high dietary and cultural value. The Mauritian palm (M. flexuosa) fruit was very high in fat $(25 \mathrm{~g} / 100 \mathrm{~g})$ and vitamin A $(763 \mu \mathrm{g} \mathrm{RE} / 100 \mathrm{~g})$, in addition to being a modest source of calcium $(74 \mathrm{mg} / 100 \mathrm{~g})$. Peach palm (B. gasipaes) was also a good source of total vitamin A (140 $\mu \mathrm{g} \mathrm{RE} / 100 \mathrm{~g})$, as well as a nutritious variation of typical cassava masato when prepared into peach palm masato $^{23}$. The high carotene value of genus Elaeis palm fruits and red palm oil $(6140 \mu \mathrm{g}$ RE/100g) has been recognised in food-based approaches to prevent hypo- vitaminosis $\mathrm{A}$ in diets emphasising tubers ${ }^{24}$. Palm heart was an important source of protein and iron; in addition, the palm tree provided thatch for housing ${ }^{25}$.

The cultivation of palm trees is related to another culturally cherished food: suri. These larvae, each weighing approximately $12 \mathrm{~g}$, are assumed to be high in protein $(34 \mathrm{~g} / 100 \mathrm{~g})$, fat $(39 \mathrm{~g} / 100 \mathrm{~g})$ and iron $(21 \mathrm{mg})$ based on food composition for beetle larvae of the same order (Coleopterus) $^{26-28}$. In 1987, Dufour studied insect consumption among the Tukanoan indigenous people of the Vaupes region of Colombia, and found these insects contributed to diet in amounts comparable with other animal foods. Men and women obtained as high as $12 \%$ and $26 \%$ respectively of their daily animal protein from insects $^{29}$. Invertebrate consumption is part of the traditional food system of 32 other Amerindian groups in the Amazon basin ${ }^{30}$.

Tukanoans included invertebrates in the diet seasonally and consumed greater quantities when fish and game were less available ${ }^{29}$. Unlike the Tukanoans, in the low fishing season invertebrates were not consumed in great quantities by the lower Cenepa Awajún. However, suri deserves attention as a means of local food security because of the cultural acceptability and high taste appeal among women and children ${ }^{25}$. Berlin and Markell (1977) reported that $58 \%$ of Awajún monthly dietary household protein came from fish ${ }^{11}$, but this was a negligible source of dietary protein for our season of heavy rain and resulting high rivers for the Awajún, which hindered local fishing methods.

Wild game had great nutritional significance for the lower Cenepa Awajún. Armadillo provided a source of dietary iron, protein, fat, zinc and vitamin A, especially for women $(29 \mathrm{~g}$ of protein, $5.4 \mathrm{~g}$ of fat, $10.9 \mathrm{~g}$ of iron and $225 \mu \mathrm{g} \mathrm{RE} / 100 \mathrm{~g})^{18}$. Other important game meats in the study were agouti, paca, coati (Procyonidae nasua nasua $)^{14}$ and collared peccary. The importance of wild meat as a source of food security and protein for people subsiding in tropical forests has been recognised ${ }^{31}$.

\section{Conclusions}

The TFDS may prove useful in assessing and promoting the potential of traditional food systems of indigenous peoples throughout the world. The value of diversity in the traditional food system to meet the nutrient needs of Awajún living in the lower Cenepa River area was reported, with emphasis on unique nutrient-dense components of the food system. Using a traditional food diversity score calculated from repeat 24-hour recalls, we demonstrated that greater diversity was associated with higher intakes of dietary protein, fibre, iron, thiamin, riboflavin and vitamin A among women and children. In addition, higher dietary calcium, phosphorus, niacin, 
vitamin C and folate were correlated with greater diversity scores for women.

Unique animal and plant foods were identified that provided outstanding nutrients for the Awajún. This biodiversity should be protected and promoted to support the Awajún right to subsistence lifestyle and good health. Not only is traditional food nutritionally beneficial to indigenous peoples, access to these resources is also a human right ${ }^{2}$. We affirm the suggestion that to deny the right to food is to deny the collective indigenous existence, ${ }^{2}$.

\section{Acknowledgements}

Sources of funding: The study was funded by the Canadian Institutes for Health Research (CIHR), Institute of Population and Public Health (IPPH), and Institute of Aboriginal Peoples Health (IAPH).

Conflict of interest declaration: There is no conflict of interest.

Authorship responsibilities: M.L.R. was a McGill MSc student at the time of this research. H.M.C.-K. was the Peruvian academic partner, and I.T. was the Awajún lead partner in the research. H.V.K. was the overall research coordinator.

Acknowledgements: We sincerely thank Sandra Vidal, Melissa Abad, Miluska Carrasco for their work in data collection. We also thank our consistently resourceful and helpful Awajún community leaders and research assistants for their efforts in translation. ODECFROC and the dedicated women's group were essential throughout the research, for which we are grateful. We would like to thank Margot Marin of the IIN for her work in data entry, and the Amazon Conservation Team (Washington, DC) for assistance with scientific identifications. We extend our appreciation to the communities of the lower Cenepa River, especially the women and children who participated in the study. We also note with appreciation funding from the CIHR, IPPH and IAPH.

\section{References}

1 Kuhnlein HV, Receveur O. Dietary change and traditional food systems of Indigenous Peoples. Annual Review of Nutrition 1996; 16: 417-42.

2 Kuhnlein HV, Erasmus B, Kalafatic C. Indigenous Peoples' consultation on the right to food: Declaration of Atitlan, Guatemala. SCN News 2002; 24: 74-5.

3 Johns T, Sthapit BR. Biocultural diversity in the sustainability of developing-country food systems. Food and Nutrition Bulletin 2004; 25: 143-55.

4 World Health Organization (WHO)/Food and Agriculture Organization of the United Nations. Preparation and Use of Food-based Dietary Guidelines. Geneva: Nutrition Programme, WHO, 1996.

5 Ruel MT. Operationalizing dietary diversity: a review of measurement issues and research priorities. Journal of Nutrition 2003; 133(Suppl.): 3911S-26S.
6 Drewnowski A, Henderson SA, Driscoll A, Rolls BJ. The dietary variety score: assessing diet quality in healthy young and older adults. Journal of the American Dietetic Association 1997; 97: 266-71.

7 Mirmiran P, Azadbakht L, Esmaillzadeh A, Azizi F. Dietary diversity score in adolescents - a good indicator of the nutritional adequacy of diets: Tehran lipid and glucose study. Asia Pacific Journal of Clinical Nutrition 2004; 13: 56-60.

8 Hatloy A, Torheim LE, Oshaug A. Food variety. A good indicator of nutritional adequacy of the diet? A case study from an urban area in Mali, West Africa. European Journal of Clinical Nutrition 1998; 52: 891-8.

9 Torheim LE, Ouattara F, Diarra MM, Thiam FD, Barikmo I, Hatloy A, et al. Nutrient adequacy and dietary diversity in rural Mali: association and determinants. European Journal of Clinical Nutrition 2004; 58: 594-604.

10 Murphy SP, Foote JA, Wilkens LR, Basiotis PP, Carlson A, White KKL, et al. Simple measures of dietary variety are associated with improved dietary quality. Journal of the American Dietetic Association 2006; 106: 425-9.

11 Berlin EA, Markell EK. An assessment of the nutritional and health status of an Aguaruna Jivaro community, Amazonas, Peru. Ecology of Food and Nutrition 1977; 6: 69-81.

12 Sistema de Información sobre Comunidades Nativas Tituladas del Perú (SICNA). Datos de Población de la Cuenca del Cenepa Provincia Condorcanqui. Departamento Amazonas. Lima: Instituto del Bien Común, 1999.

13 Basiotis PB, Welsh SO, Cronin FJ, Kelsay JL, Mertz W. Number of days of food intake records required to estimate individual and group nutrient intakes with defined confidence. Journal of Nutrition 1987; 117: 1638-41.

14 Cypel YS, Guenther PM, Petot GJ. Validity of portion-size measurement aids: a review. Journal of the American Dietetic Association 1997; 97: 289-92.

15 US Department of Agriculture. Agricultural Research Service Nutrient Data Laboratory Home Page [online], 2004. Available at http://www.nal.usda.gov/fnic/food$\mathrm{comp} / \mathrm{search} /$. Accessed?.

16 Wu Leung WT, Butrum RR, Chang FH, Rao MN, Polacchi W. Food Composition Table for Use in East Asia. Rome/ Washington, DC: Food and Agriculture Organization of the United Nations/US Department of Health, Education, and Welfare, 1972.

17 Wu Leung WT, Flores M. INCAP-ICNND Food Composition Table for Use in Latin America. Guatemala City/Bethesda, MD: Institute of Nutrition of Central America and Panama/ National Institutes of Health, 1961.

18 Herrera NA, Hernández FA, Fachin RN, Robles NG. La Composición de los Alimentos Autoctonos. Lima: Laboratorios de Bromatología y Bioquímica, Instituto de Nutrición, Institutos Nacionales de Salud and Ministerio de Salud, undated.

19 Sims J, Kuhnlein HV. Indigenous Peoples Participatory Health Research. Planning and Management. Preparing Research Agreements. Geneva: World Health Organization, 2003.

20 Kye S, Lee H, Kim BH, Yoo W, Sung MK, Lee HJ. Dietary variety and nutrient intake by 24-hour recall in Korean college students. Nutritional Sciences 2004; 7: 92-7.

21 Maunder EMW, Matji J, Hlatshwayo-Molea T. Enjoy a variety of foods - difficult but necessary in developing countries. South African Journal of Clinical Nutrition 2001; 14: $S 7-11$.

22 Roche ML, Creed-Kanashiro HM, Tuesta I, Kuhnlein HV. Traditional food system provides dietary quality for the Awajún in the Peruvian Amazon. Ecology of Food and Nutrition 2007; in press.

23 Instituto de Investigación Nutritcional (IIN). Tabla de Composición de Alimentos. Lima: IIN, 2004. 
24 Solomons NW, Orozco M. Alleviation of vitamin A deficiency with palm fruit and its products. Asia Pacific Journal of Clinical Nutrition 2003; 12: 373-84.

25 Brown MF. Una paz incierta, historia y cultura de las comunidades aguarunas frente al impacto de la carretera marginal. Lima: Centro Amazonico de Antropologia y Aplicacion Pratica, 1984.

26 Ramos-Elorduy J, Pino Moreno JM, Escamilla Prado E, Alvarado Perez M, Lagunez Otero J, Ladron de Guevara O. Nutritional value of edible insects from the State of Oaxaca, Mexico. Journal of Food Composition and Analysis 1997; 10: $142-57$.

27 Finke MD. Complete nutrient composition of commercially raised invertebrates used as food for insectivores. ZOO Biology 2002; 21: 269-85.
28 Paoletti MG, Buscardo E, Vander Jagt DJ, Pastuszyn A, Pizzoferrato L, Huang YS, et al. Nutrient content of earthworms consumed by Ye'Kuana Amerindians of the Alto Orinoco of Venezuela. Proceedings of the Royal Society 2003; 270: 249-57.

29 Dufour DL. Insects as food: a case study from the northwest Amazon. American Anthropologist 1987; 89: 383-96.

30 Paoletti MG, Dufour DL, Cerda H, Torres F, Pizzoferrato L, Pimentel D. The importance of leaf- and litter-feeding invertebrates as sources of animal protein for the Amazonian Amerindians. Proceedings of the Royal Society 2000; 267: 2247-52.

31 Bennett EL. Is there a link between wild meat and food security? Conservation Biology 2002; 16: 590-2. 